\title{
New Dynamic Equations of Aerosol in Air of Certain Type
}

\author{
Tian-Quan Yun \\ School of Civil Engineering and Transportation, South China University of Technology, Guangzhou, China \\ Email: cttqyun@scut.edu.cn
}

How to cite this paper: Yun, T.-Q. (2017)

New Dynamic Equations of Aerosol in Air of Certain Type. Atmospheric and Climate Sciences, 7, 511-524.

https://doi.org/10.4236/acs.2017.74037

Received: August 17, 2017

Accepted: October 7, 2017

Published: October 10, 2017

Copyright $\odot 2017$ by author and Scientific Research Publishing Inc. This work is licensed under the Creative Commons Attribution International License (CC BY 4.0).

http://creativecommons.org/licenses/by/4.0/

\begin{abstract}
A new dynamic equation of aerosol in air is derived, using a model-in-model, by equilibrium of buoyancy, gravity and pressure, together with conservation laws of mass, momentum and energy via Reynolds transport theorem and supplemented by corresponding scientific laws for related properties of air and aerosols. This new dynamic equation of aerosol in air is a set of non-linear partial differential equations involved six unknown functions of mass densities, pressure, air and aerosol speeds and temperature. It has features: 1, it belongs to certain type; 2, it emphases the effect of buoyancy in equilibrium and potential energy, and the Archimedes principle of buoyancy is firstly extended to lateral directions based on logical deduction, the phenomenon of stirring a glass of oil-water mixture and the recorded of Hurricane Isabel (2003) from space station. The later shows the evidence of existence of lateral buoyancy; 3, the mass densities of air and aerosol of a point in our model are varied in different directions due to traction and are treated as vectors, and they have been used in the calculation of lateral buoyancy.
\end{abstract}

\section{Keywords}

Aerosol, Vapor, Condensation, Coagulation, Diffusion, Boyle's Law, Charles' Law, Newton's Law, Archimedes Principle of Buoyancy, Reynolds Transport Theorem, Wind Speed Equation

\section{Introduction}

An aerosol includes solid particles or liquid droplets in suspending air. Various types of aerosol, such as dust, fume, smoke, cloud, fog, haze, air pollutants, PM 2.5, PM 10, etc., have various physical and chemical properties related to corresponding scientific laws. However, their motion in air can be described by a general governing dynamic equation of aerosol in air with certain type (determi- 
nistic type).

There are two kinds of methods: one of them belongs to the "certain type", where a problem is expressed by differential equation(s) (integral equation, etc.) with initial, boundary value(s), its solution is certain; the other belongs to the "uncertain type", where a problem (usually involves random, or stochastic factor) is reduced to expression based on statistic, probability, etc. its solution is uncertain, or the rule it obtained is certain only under the sense of statistic.

The general governing dynamic equation of aerosols has already formulated ([1] [2] [3]) as:

$\frac{\partial n(v, r, t)}{\partial t}+\nabla[u(v, r, t) n(v, r, t)]-\nabla[D(v, r, t)-\nabla n(v, r, t)]$
$=-[(\partial n(v, r, t)) / \partial t]_{\text {growth }}-[(\partial n(v, r, t)) / \partial t]_{\text {removal }}-[(\partial n(v, r, t)) / \partial t]_{\text {coag }}+S(v, r, t)$

where $n(v, r, t)$ is the number of aerosol particles per unit volume $v$, in point $r$, at time $t, u(v, r, t)$ is the vector sum of the air and aerosol particles velocity; $D(v, r, t)$ is the Brownian diffusion coefficient; $\nabla$ is del operator; the right side of (1-1) represent the time rate of $n$ due to growth, removal, coagulation respectively; $S(v, r, t)$ is an independent source term.

The above governing dynamic equation has at least three lacks in perfect: it involves random factor, e.g., the Brownian motion assumption, and leads to an uncertain type results; it neglects buoyancy effect as usually the calculation of terminal velocity of a particle in a fluid [4]; it complicates the effect of distribution of number of particles, size, and shapes on suspension motion.

The purpose of this paper is to set up a general governing dynamic equation of aerosol of certain type. In which, no random factors like Brownian motion assumption have been involved. Furthermore, it respects Archimedes principle of buoyancy as well as Newton's gravity law in vertical motion calculation, and the Archimedes principle of buoyancy is firstly extended from the z-direction to lateral directions. And the calculation of the two-phased motion of suspension aerosols and air is simplified by the one-phased motion of air, at least, in which the "wind speed equation" [5] and its solution [6] for the Cartesian coordinates, the "wind speed equation of circular cyclone" [7] and its solution [8] for cylindrical coordinates have been found.

Section 2, a model-in-model is established and the densities of aerosol and air are defined as vectors. In Section 3, the importance of Archimedes principle of buoyancy is emphasized and is extended to lateral directions. In Section 4, the Reynolds transport theorem is mentioned. In Section 5, conservation laws of mass, momentum and energy of aerosol in air have been set up, in which the potential energy is defined by adding potential energy due to buoyancy to the traditional definition of potential energy due to gravity. In Section 6, the dynamic equation of aerosol in air has been set up by equilibrium of body forces, buoyancy and gravity force, and pressure. In Section 7, unknown variables linked with corresponding scientific laws for air and some aerosols are mentioned. Summing up the results of Section 5, 6, and 7, we get the general go- 
verning dynamic equations of aerosol in air. It is a set of nonlinear differential vector equations with six unknown functions $\rho_{s}, \rho_{a}, u_{s}, u_{a}, p$ and $T$ shown in Section 8. In Section 9, comparisons have been made. In which the features have been pointed. Finally, a conclusion is made.

\section{Notation, Definition and Model-in-Model}

The model of a box wrapped by "zero-weighted membrane" had been used to set up a "wind speed equation in air" for Cartesian coordinates [5], and the model of a cylinder wrapped by "zero-weighted membrane" had been used to set up "wind speed equation of circular cyclone" for cylindrical coordinates [7]. Here, a model-in-model is used to set up a new dynamic equation for the two-phased motion problem of aerosol in air.

\subsection{A Model-in-Model Wrapped by "Zero-Weighted Membrane"}

A box involved air, with surfaces $A_{x}=\mathrm{d} y \mathrm{~d} z, A_{y}=\mathrm{d} z \mathrm{~d} x, A_{z}=\mathrm{d} x \mathrm{~d} y$ and volume $V=\mathrm{d} x \mathrm{~d} y \mathrm{~d} z$ wrapped by a "zero-weighted membrane", and a sub-model involved aerosol with arbitrary "control volume" $\left(V_{s}\right)$, smooth "control surface" $\left(S_{s}\right)$, wrapped by "zero-weighted membrane", as $\mathrm{d} x \rightarrow 0, \mathrm{~d} x \rightarrow 0$ and $\mathrm{d} z \rightarrow 0$, is called a "point" (or a box) in air.

When the weight of the membrane of the box approaches to zero, that means nothing wrapped the box, or the box is wrapped by an imagine membrane. It does not change the actual field to be studied. However, such a box has shape, boundary and volume, and thus it can be used to set up equations as [5] [7].

In the following, a point $N(x, t)$ is measured by a fixed referent frame in Cartesian coordinates with $z=0$ at sea level and $z>0$ for upward.

$\boldsymbol{x}=(x, y, z)=x \boldsymbol{i}+y \boldsymbol{j}+z \boldsymbol{k}$, where $\boldsymbol{i}, \boldsymbol{j}, \boldsymbol{k}$ are unit vectors, along with the $\mathrm{x}$-axis, $\mathrm{y}$-axis, and $\mathrm{z}$-axis respectively, $t$ is the time. The bold face denotes vector. The symbol " $A:=B$ " means $A$ is defined by $B$.

Note that $V=V(x, t)$ (not a constant).

The volume of a point involves air and aerosol, i.e.,

$$
V=V_{a}+V_{s},
$$

where subscript a denotes the volume belonging to air; subscript $s$ denotes the volume belonging to aerosol. The volume $V_{s}$ is involved in air of the point with closed smooth surface $S_{s}\left(V_{s}\right.$ and $S_{s}$ are called control volume and control surface in fluid mechanics, respectively). Note that the volume $V_{s}=V_{s}(\boldsymbol{x}, t)$ represents the volumes of summation of total particles, with different sizes, shapes, of an aerosol (such as fog, cloud, dust, haze, PM2.5. PM10, etc.) or the mixture of them, in the point.

\subsection{The Mass and Density of Aerosol in Air}

The densities of mass of air and aerosol in the point are defined in (2-2) and (2-3) respectively. 


$$
\begin{gathered}
\boldsymbol{\rho}_{a}=\boldsymbol{\rho}_{a}(\boldsymbol{x}, t)=\boldsymbol{m}_{a} / V, \\
\boldsymbol{\rho}_{s}=\boldsymbol{\rho}_{s}(\boldsymbol{x}, t)=\boldsymbol{m}_{s} / V, \\
\boldsymbol{m}=\boldsymbol{m}_{s}+\boldsymbol{m}_{a}, \\
\boldsymbol{\rho}=\boldsymbol{\rho}_{s}+\boldsymbol{\rho}_{a},
\end{gathered}
$$

$\boldsymbol{m}, \boldsymbol{\rho}, \boldsymbol{m}_{a}$ and $\boldsymbol{m}_{s}$ are the mass of the point, density of the point, mass of the air and mass of aerosol of the point, respectively.

There are two measures of aerosol mass concentration, one is mass per unit volume with units, such as $\left(\mu \mathrm{g} / \mathrm{m}^{3}\right)$; the other is the number of particles per unit volume. We use the first one, i.e., (2-2), (2-3), since the number of particles leads to un-certain analysis.

\subsection{Extension the Mass and Density to Vectors of Function of Space and Time}

\section{Discussion: mass $m$ is a scalar or a vector?}

It depends on the model involved the mass.

If $m:=\left\{\int_{0}^{V} s \mathrm{~d} V \mid V\right.$ is wrapped by rigid walls $\}$, then, no $s$ (substance or particle) can move in or out of the walls along any direction, i.e., $m$ keeps constant and $m$ is a scalar, e.g., the mass of a solid.

If $m:=\left\{\int_{0}^{V} s \mathrm{~d} V \mid V\right.$ is wrapped by zero-weighted membrane $\}$, then, $s$ can move in or out of the membrane due to traction and is varied with directions. Therefore $m$ should be treated as a vector.

Or, a scalar is characterized as that it has no components.

$$
\{m=\text { scalar }\} \leftrightarrow\{\boldsymbol{m}=-\boldsymbol{m}\} \leftrightarrow\{\boldsymbol{m}=\mathbf{0}\}
$$

$\leftrightarrow\left\{m_{x}=(\boldsymbol{m} \cdot \mathbf{i})=(\mathbf{0} \cdot \boldsymbol{i})=0, m_{y}=0, m_{z}=0\right\}$, where $(\boldsymbol{m} \cdot \boldsymbol{i})$ is the inner product of $m$ and $i$.

Here, the $\boldsymbol{m}_{a}$ and $\boldsymbol{m}_{s}$ are defined as vectors of function of space $\boldsymbol{x}$ and time $t$, due to condensation, evaporation, coagulation, diffusion, chemical reaction, etc. Because $\boldsymbol{m}, \boldsymbol{\rho}, \boldsymbol{m}_{a}$ and $\boldsymbol{m}_{s}$ have its non-zero components therefore they should be described by vectors.

The density component of air $\rho_{a x}$ is recognized to be varied with altitude $z$, due to gravity acceleration $g$, i.e., $\rho_{a z}=\rho_{a z}(z)$.

Similarly, we extend this concept to the lateral $\mathrm{x}$-axis and $\mathrm{y}$-axis for acceleration $\boldsymbol{a}_{x}$ and $\boldsymbol{a}_{y}$, respectively by logical deduction, i.e., $\rho_{a x}=\rho_{a x}(x, t)$, $\rho_{a y}=\rho_{a y}(x, t), \rho_{a z}=\rho_{a z}(x, t)$. Thus $\rho_{a}=\left(\rho_{a x}, \rho_{a y}, \rho_{a z}\right)$ is a vector. Similarly, for aerosol $\rho_{\mathrm{s}}$, we have:

$$
\begin{aligned}
& \rho_{s x}=\frac{m_{s x}}{V}=\rho_{s x}(x, t), m_{s x}=m_{s} \cdot \cos \alpha, \\
& \rho_{s y}=\frac{m_{s y}}{V}=\rho_{s y}(x, t), m_{s y}=m_{s} \cdot \cos \beta,
\end{aligned}
$$




$$
\rho_{s z}=\frac{m_{s z}}{V}=\rho_{s z}(x, t), m_{s z}=m_{s} \cdot \cos \gamma,
$$

where $\alpha, \beta, \gamma$ are the angles between vector $\rho_{\mathrm{s}}$ and the x-axis, y-axis, and $\mathrm{z}$-axis respectively.

$$
\boldsymbol{\rho}_{s}=\sqrt{\rho_{s x}^{2}+\rho_{s y}^{2}+\rho_{s z}^{2}}=\boldsymbol{m}_{s} / V
$$

\subsection{Position $x_{a}, x_{s}$, Velocity $u_{a}, u_{s}$, Acceleration $a_{a}, a_{s}$ and Relative Velocity $\delta u$, Relative Acceleration $\delta a$.}

Since the motions of air and aerosol of a point may have its own traces. The notation $\boldsymbol{x}_{a}$ and $\boldsymbol{x}_{s}$ denote the spatial positions of air and aerosol respectively. $\boldsymbol{u}_{a}=\boldsymbol{u}_{a}\left(\boldsymbol{x}_{a}, t\right)=\frac{\mathrm{d} \boldsymbol{x}_{a}}{\mathrm{~d} t}=\frac{\partial \boldsymbol{x}_{a}}{\partial t}+\left(u_{x} \boldsymbol{i}+u_{y} \boldsymbol{j}+u_{z} \boldsymbol{k}\right)$ and

$$
\boldsymbol{u}_{s}=\boldsymbol{u}_{s}\left(\boldsymbol{x}_{s}, t\right)=\frac{\mathrm{d} \boldsymbol{x}_{s}}{\mathrm{~d} t}=\frac{\partial \boldsymbol{x}_{s}}{\partial t}+\left(u_{x} \boldsymbol{i}+u_{y} \boldsymbol{j}+u_{z} \boldsymbol{k}\right)
$$

represent the velocity at the point of air and of aerosol, respectively.

$$
\delta \boldsymbol{u}=\boldsymbol{u}_{a}-\boldsymbol{u}_{s}=\partial\left(\boldsymbol{x}_{a}-\boldsymbol{x}_{s}\right) / \partial t
$$

Equation (2-10) represents the relative velocity between point air and point aerosol. If $\delta \boldsymbol{u}=\mathbf{0}$, or $\boldsymbol{u}_{a}=\boldsymbol{u}_{s}=\boldsymbol{u}$, that means the aerosol moving along with the trace of air. If $\delta \boldsymbol{u} \neq \mathbf{0}$, e.g., the vapor condenses into water droplet and forms rain, then, $\boldsymbol{u}_{a} \neq \boldsymbol{u}_{s}$.

$$
\begin{gathered}
\boldsymbol{a}_{a}=\frac{\mathrm{d} \boldsymbol{u}_{a}}{\mathrm{~d} t}=\frac{\partial \boldsymbol{u}_{a}}{\partial t}+\frac{\partial u_{a}}{\partial x_{a}} \frac{\partial x_{a}}{\partial t}+\frac{\partial u_{a}}{\partial y_{a}} \frac{\partial y_{a}}{\partial t}+\frac{\partial u_{a}}{\partial z_{a}} \frac{\partial z_{a}}{\partial t} \\
=\frac{\partial \boldsymbol{u}_{a}}{\partial t}+\frac{\partial u_{a}}{\partial x_{a}} u_{a x}+\frac{\partial u_{a}}{\partial y_{a}} u_{a y}+\frac{\partial u_{a}}{\partial z_{a}} u_{a z} \\
=\frac{\partial \boldsymbol{u}_{a}}{\partial t}+\boldsymbol{u}_{a} \cdot \nabla \boldsymbol{u}_{a} \\
\boldsymbol{a}_{s}=\frac{\partial \boldsymbol{u}_{s}}{\partial t}+\boldsymbol{u}_{s} \cdot \nabla \boldsymbol{u}_{s}, \\
\boldsymbol{u}=\left(u_{x}, u_{y}, u_{z}\right)=\left(\frac{\partial x}{\partial t}, \frac{\partial y}{\partial t}, \frac{\partial z}{\partial t}\right), \\
\nabla \boldsymbol{u}=i \frac{\partial}{\partial x} u_{x}+j \frac{\partial}{\partial y} u_{y}+\boldsymbol{k} \frac{\partial}{\partial x} u_{z}, \\
\delta \boldsymbol{a}=\boldsymbol{a}_{a}-\boldsymbol{a}_{s}=\frac{\partial \delta \boldsymbol{u}}{\partial t}+\boldsymbol{u}_{a} \cdot \nabla \boldsymbol{u}_{a}-\boldsymbol{u}_{s} \cdot \nabla \boldsymbol{u}_{s} .
\end{gathered}
$$

Equations (2-11), (2-12) and (2-15) represent the acceleration of air, aerosol and relative acceleration of the point respectively.

\section{Buoyancy}

Archimedes's principle states that any object, wholly or partly, immersed in a fluid, is buoyed by a force equal to the weight of the fluid displaced by the object. The Archimedes's principle used here in atmospheric calculation has 3 features: 
1, emphases its importance, it can not be neglected; 2, Archimedes's principle is originally stated in vertical balance, and is now generalized to horizontal directions; 3 , the mass density of aerosol and air are treated as vector.

\subsection{Action and Reaction are Equal Important for Gravity and Buoyancy}

The Newton's third law: action and reaction forces are equal in value and opposite in direction, also suits for Newton's gravity law and Archimedes' principle of buoyancy.

The Archimedes principle of buoyancy, can be viewed as an opposite law of Newton's gravity law. When an aerosol suspense in air, it shows that the applied forces on aerosol is in equilibrium, i.e., the gravity force equals to the buoyancy force.

Both sides of action and reaction are equally important in natural and society world. If only Newton's gravity law holds, without Archimedes' principle of buoyancy works as balance or neglecting the influence of buoyancy [4], then, all objects with mass would be attracted to the Earth. The Earth becomes death. The Earth being a planet with life is relying on the gravity force and buoyancy force, the later makes cycles of water to evaporation to cloud, cloud to water droplet, and water droplet to rain. The cycles brings water to everywhere on Earth to keep life existence.

\subsection{Extension the Archimedes' Principle of Buoyancy to Lateral Coordinates}

The Archimedes' principle of buoyancy

$$
F_{b z}=g V\left(\rho_{a z}-\rho_{s z}\right),
$$

is originally established for vertical motion, in z-direction, against gravity force, and now is firstly extended to lateral motion, in the $\mathrm{x}$-axis and the $\mathrm{y}$-axis direction. i.e., similarly to (3-1), by logical deduction, which assumes that a rule suits for the $\mathrm{z}$-axis is also suited for the $\mathrm{x}$-axis and the $\mathrm{y}$-axis, then we have:

$$
\begin{aligned}
& F_{b x}=V\left(a_{a x} \rho_{a x}-a_{s x} \rho_{s x}\right), \\
& F_{b y}=V\left(a_{a y} \rho_{a y}-a_{s y} \rho_{s y}\right),
\end{aligned}
$$

where $a_{a x}, a_{a y}$ and $a_{s x}, a_{s y}$ are components of accelerations $\boldsymbol{a}_{a}, \boldsymbol{a}_{s}$ in the $\mathrm{x}$-axis and the $\mathrm{y}$-axis direction respectively.

Such an extension is based on logical deduction, the phenomenon of stirring a glass of oil-water mixture and a recorded photo of Hurricane Isabel (2003). When an oil-water in a glass is stirring to make the lateral acceleration occurring, then, the lighter substance, oil level, move from boundary to the centre, this phenomenon shows the existence of lateral buoyancy. The clear "eye", free of clouds, recorded of Hurricane Isabel (2003) from space station [9] also shows the evidence of existence of lateral buoyancy in nature, where lighter density substances move to the centre (eye) of the cyclone due to lateral buoyancy. 


\section{Reynolds Transport Theorem}

The Reynolds transport theorem is a basic theorem used in formulating basic conservation laws of fluid [10]. It can also be viewed as a 3-D generalization of Leibniz integral rule, which is also known as differentiation under integral sign [11]. It studies the changing of the order of differentiation and integration operators.

One form of the Reynolds transport theorem [11] is:

$$
\begin{aligned}
& \frac{\partial}{\partial t}\left(\iiint_{V_{s}(t)} f(\boldsymbol{x}, t) \mathrm{d} V_{s}\right) \\
& =\iiint_{V_{s}(t)} \frac{\partial f(\boldsymbol{x}, t)}{\partial t} \mathrm{~d} V_{s}+\iint_{S_{s}(t)} f(\boldsymbol{x}, t)(\boldsymbol{v} \cdot \boldsymbol{n}) \mathrm{d} S_{s}
\end{aligned}
$$

where $f(\boldsymbol{x}, t)$ represents an arbitrary function of space $\boldsymbol{x}$ and time $t . \quad \boldsymbol{v}$ is velocity of the fluid passing through $S_{s} \cdot \nabla$ is the del operator. $\boldsymbol{n}$ is the outward pointing normal unit vector on $S_{s}$. The left side of (4-1) represents time rate of total $f(x, t)$ in $V_{s}$. The first term of the right side of (4-1) represents the total time rate of $f(x, t)$ of points in $V_{s}$ and the second term represents the net flux of $f(x, t)$ passing through $S_{s}$.

The Reynolds transport theorem is used to derive conservation laws of aerosol in air.

\section{Conservation Laws of Aerosol in Air}

\subsection{Conservation of Mass}

Let

$$
f(x, t)=\rho_{s}(x, t)=\boldsymbol{m}_{s} / V
$$

Substituting (5-1) into (4-1), and $\boldsymbol{v}=\delta \boldsymbol{u}=\boldsymbol{u}_{a}-\boldsymbol{u}_{s}$, then the conservation law of mass is:

$$
\begin{aligned}
0 & =\frac{\partial m_{s}}{\partial t}=\frac{\partial}{\partial t} \iiint_{V_{s}(t)} \boldsymbol{\rho}_{s}(\boldsymbol{x}, t) \mathrm{d} V_{s} \\
& =\iiint_{V_{S}(t)} \frac{\partial \boldsymbol{\rho}_{s}(\boldsymbol{x}, t)}{\partial t} \mathrm{~d} V_{s}+\iint_{S_{s}(t)} \boldsymbol{\rho}_{s}(\boldsymbol{x}, t)(\delta \boldsymbol{u} \cdot \boldsymbol{u}) \mathrm{d} S_{s}
\end{aligned}
$$

Equation (5-2) is called the conservation law of mass of aerosol. It shows that the total changes of density $\rho_{s}$ in $V_{s}$ equals to the net flux of $\rho_{s}$ passing through the surface $S_{s}$.

By the theorem of vector integration (Gauss formula [12]), (it is also called the divergent theorem)

$$
\iiint_{V_{s}} \nabla L \mathrm{~d} V_{s}=\iint_{S_{s}} L \cdot n \mathrm{~d} S_{s} .
$$

Substituting $L=f \cdot \delta \mathbf{u}$ (5-1), (5-3) into (5-2), we have

$$
\iiint_{V_{s}}\left[\frac{\partial \boldsymbol{\rho}_{s}}{\partial t}+\nabla \cdot\left(\boldsymbol{\rho}_{s} \delta \mathbf{u}\right)\right] \mathrm{d} V_{s}=0
$$

Equation (5-4) holds for arbitrary volume $V_{s}$, then, we have: 


$$
\begin{aligned}
& \frac{\partial \boldsymbol{\rho}_{s}}{\partial t}+\nabla\left(\boldsymbol{\rho}_{s} \delta \boldsymbol{u}\right)=0,(\text { for } \delta u \neq 0) \\
& \frac{\partial \boldsymbol{\rho}_{s}}{\partial t}+\nabla\left(\rho_{s} \boldsymbol{u}\right)=0,(\text { for } \delta u=0)
\end{aligned}
$$

Equation (5-5) shows that the time rate of density $\rho_{s}$ is equal to the minus space rate $\nabla$ of $\left(\boldsymbol{\rho}_{s} \delta \boldsymbol{u}\right)$ in a point. It is a differential form of conservation law of mass for aerosol.

Similarly, we have:

$$
\begin{aligned}
& \frac{\partial \boldsymbol{\rho}_{a}}{\partial t}+\nabla\left(\boldsymbol{\rho}_{a} \delta \boldsymbol{u}\right)=0,(\text { for } \delta u \neq 0) \\
& \frac{\partial \boldsymbol{\rho}_{a}}{\partial t}+\nabla \cdot\left(\boldsymbol{\rho}_{a} \boldsymbol{u}\right)=0,(\text { for } \delta u=0)
\end{aligned}
$$

Equation (5-6) is the conservation law of mass of air.

\subsection{Conservation Law of Momentum of Aerosol in Air}

Let

$$
f(\boldsymbol{x}, t)=\left[\rho_{s} \boldsymbol{u}_{s}\right],
$$

Substituting $f$ of (5-7) into (4-1), we have

$$
\begin{aligned}
& \frac{\partial}{\partial t} \iiint_{V_{s}(t)}\left[\boldsymbol{\rho}_{s} \boldsymbol{u}_{s}\right] \mathrm{d} V_{s} \\
& =\iiint_{V_{s}(t)}\left[\boldsymbol{\rho}_{s} \frac{\partial \boldsymbol{u}_{s}}{\partial t}+\boldsymbol{u}_{s} \frac{\partial \boldsymbol{\rho}_{s}}{\partial t}\right] \mathrm{d} V_{s}+\iint_{S_{s}(t)}\left[\boldsymbol{\rho}_{s} \boldsymbol{u}_{s}\right](\delta \boldsymbol{u} \cdot \boldsymbol{n}) \mathrm{d} S_{s}=0
\end{aligned}
$$

Substituting (5-8) into (5-3), change the surface integral to volume integral, then (5-8) becomes:

$$
\begin{gathered}
\iiint_{V_{s}(t)}\left[\frac{\partial\left[\boldsymbol{\rho}_{s} \boldsymbol{u}_{s}\right]}{\partial t}+\nabla \cdot\left[\left(\boldsymbol{\rho}_{s} \boldsymbol{u}_{s}\right) \delta \mathbf{u}\right]\right] \mathrm{d} V_{s}=0, \\
\frac{\partial\left[\boldsymbol{\rho}_{s} \boldsymbol{u}_{s}\right]}{\partial t}+\nabla \cdot\left[\left(\boldsymbol{\rho}_{s} \boldsymbol{u}_{s}\right) \delta \boldsymbol{u}\right]=0, \quad(\text { for } \delta \boldsymbol{u} \neq 0) \\
\frac{\partial\left[\boldsymbol{\rho}_{s} \boldsymbol{u}_{s}\right]}{\partial t}+\nabla \cdot\left[\left(\boldsymbol{\rho}_{s}\right) \boldsymbol{u} \cdot \boldsymbol{u}\right]=0, \quad(\text { for } \delta \boldsymbol{u}=0)
\end{gathered}
$$

Similarly, we have

$$
\begin{aligned}
& \frac{\partial\left[\rho_{a} \boldsymbol{u}_{a}\right]}{\partial t}+\nabla \cdot\left[\left(\boldsymbol{\rho}_{a} \boldsymbol{u}_{a}\right) \delta \boldsymbol{u}\right]=0, \quad(\text { for } \delta \boldsymbol{u} \neq 0) \\
& \frac{\partial\left[\boldsymbol{\rho}_{a} \boldsymbol{u}_{a}\right]}{\partial t}+\nabla \cdot\left[\left(\boldsymbol{\rho}_{a}\right) \boldsymbol{u} \cdot \boldsymbol{u}\right]=0, \quad(\text { for } \delta \boldsymbol{u}=0)
\end{aligned}
$$

Equation (5-11) is called the conservation law of momentum of air.

\subsection{Conservation Law of Energy of Aerosol and Air in a Point}

Different from the traditional concept of potential energy $E_{p}$, in which $E_{p}$ is defined by the work done gravity force on altitude displacement, while herein, 
$E_{p}$ is defined as the work done by the sum of body (including gravity) force and buoyancy on displacements of three components, i.e., the $x$-axis, $y$-axis and $z$-axis, as shown in following.

Let

$$
\begin{gathered}
E_{k}=E_{k}(\boldsymbol{x}, t)=\frac{1}{2}\left[\boldsymbol{u}_{a}^{2}+\boldsymbol{u}_{s}^{2}\right], \\
E_{p}:=E_{p}(\boldsymbol{x}, t)=\left(\rho_{s x}-\rho_{a x}\right) a_{x} \delta x+\left(\rho_{s y}-\rho_{a y}\right) a_{y} \delta y \\
+\left(\rho_{s z}-\rho_{a z}\right) g \delta z, \quad\left(\text { for } \delta \boldsymbol{a}=\mathbf{0} \text { or } \boldsymbol{u}_{a}=\boldsymbol{u}_{s}=\boldsymbol{u}\right) \\
E_{p}:=\left(\rho_{s x} a_{s x}-\rho_{a x} a_{a x}\right) \delta x+\left(\rho_{s y} a_{s y}-\rho_{a y} a_{a y}\right) \delta y \\
+\left(\rho_{s z}-\rho_{a z}\right) g \delta z, \quad\left(\text { for } \delta \boldsymbol{a} \neq \mathbf{0} \text { or } \boldsymbol{u}_{a} \neq \boldsymbol{u}_{s}\right)
\end{gathered}
$$

Substituting $f(x, t)=\delta E$ of (5-16) into $f(x, t)=\left(\rho_{s} u_{s}\right)$ of $(5-10),(5-10)_{\mathrm{A}}$, we have:

$$
\begin{aligned}
& \frac{\partial(\delta E)}{\partial t}+\nabla \cdot[\delta E \cdot \delta \boldsymbol{u}]=0,(\text { for } \delta \boldsymbol{u} \neq 0) \\
& \frac{\partial(\delta E)}{\partial t}+\nabla \cdot[\delta E \cdot \boldsymbol{u}]=0,(\text { for } \delta \boldsymbol{u}=0)
\end{aligned}
$$

where $E, E_{k}$ and $E_{p}$ are the total energy, kinetic energy and potential energy per unit volume of a point respectively; $\delta$ is an increase; $U$ is the internal energy, the change $\delta U$ of $U$ due to the work $W$ and heat $Q$ put into or out from the point. Equation (5-17) is the differential form of conservation law of energy of aerosol and air of a point.

\section{Dynamic Equation of a Point of Aerosol in Air}

We consider the equilibrium of the box shown in Section 2.1 . $\sum F_{z}=0$, we have

$$
\left[\rho_{a}-\rho_{s}\right] V g-p_{z}(x, y, z+\delta z) A_{z+\delta z}+p_{z}(x, y, z) A_{z}=0 .
$$

Expanding $p_{z}(x, y, z+\delta z)$ into Taylor expansion and keeps two terms, then, (6-1) becomes:

$$
\left[\rho_{a z}-\rho_{s z}\right] g=\frac{\partial p_{z}}{\partial z}
$$

$\sum F_{x}=0$, similarly we have

$$
\left[\rho_{a x} a_{a x}-\rho_{s x} a_{s x}\right]=\frac{\partial p_{x}}{\partial x} .
$$

$\sum F_{y}=0$, similarly we have 


$$
\left[\rho_{a y} a_{a y}-\rho_{s y} a_{s y}\right]=\frac{\partial p_{y}}{\partial y} .
$$

Equations (6-2)-(6-4) are the components expression of dynamic equation of a point of aerosol in air, in which the left-hand-side represents the difference of body forces due to buoyancy and gravity in directions of the z-axis, the $\mathrm{x}$-axis and the y-axis respectively. $a_{a x}, a_{a y}, g$ and $a_{s x}, a_{s y}, g$ are the components of accelerations $\boldsymbol{a}_{a}$ and $\boldsymbol{a}_{s}$ respectively. The right-hand-side represents the gradient of pressure components $p_{x}, p_{y}, p_{z} . \boldsymbol{p}=\left(p_{x}, p_{y}, p_{z}\right)$.

Summing up (6-2)-(6-4), we have:

$$
\rho_{a} \boldsymbol{a}_{a}-\boldsymbol{\rho}_{\mathrm{s}} \boldsymbol{a}_{s}=\nabla \cdot \boldsymbol{p} .
$$

Equation (6-5) is the vector form of dynamic equation of a point of aerosol in air.

If $\boldsymbol{\rho}_{s}=\mathbf{0}$, i.e., there is no aerosol, then (6-5) is reduced to the wind speed equation of a point in air [5].

In the above derivation, the friction or the viscosity of air is neglected.

\section{Variables Linked by Scientific Laws}

\subsection{For Air}

\subsubsection{The Combination of Boyles Law and Charles Law}

$$
p V=R T,
$$

where $p=p(x, t)$ is the pressure; $V=V(x, t)$ is the volume; $T=T(x, t)$ is temperature; $R$ is constant. From (7-1), we have:

$$
\frac{\partial p}{\partial x} V+\frac{\partial V}{\partial x} p=\frac{\partial p}{\partial x} V=R \frac{\partial T}{\partial x}
$$

where $\partial V / \partial x \rightarrow 0$ as $\mathrm{d} x \rightarrow 0, \mathrm{~d} x \rightarrow 0$ and $\mathrm{d} z \rightarrow 0$. Similarly, we have:

$$
\begin{aligned}
& \frac{\partial p}{\partial y} V=R \frac{\partial T}{\partial y}, \\
& \frac{\partial p}{\partial z} V=R \frac{\partial T}{\partial z},
\end{aligned}
$$

Equations (7-1)-(7-4) are relations between pressure $p$, volume $V$ and temperature $T$. These equations show that they are independent with time, i.e., they hold for any time.

\subsubsection{The Ideal Gas Law}

The ideal gas law was first state by Clapeyrong, E in 1834, as a combination of Boyle's law, Charles' law, and Avogadro's law [13], the common form of this law is:

$$
p V=n R_{g} T,
$$

where $p, V, T$ is the same as above; $n$ is the number of moles. $R_{g}$ is gas constant, equal to the product of Bolzmann constant and Avogadro constant. (7-5) can be derived by empirically or by kinetic theory under some assumptions in- 
cluding moles random motion etc. The element of the ideal gas law is mole or atom, its size belongs to micro-level. If one tries to use a micro-level law with random motion assumption to a problem of macro-level, then, even if one got a result, the result must belong to uncertain type. Therefore, we do not use the ideal gas law while prefer to use the combination of Boyles law and Chares law.

\subsection{For Aerosol}

Different aerosols have different properties, related to different scientific laws. Properties of some aerosols are mentioned here.

\section{Condensation and Evaporation}

Condensation of mass causes the mode of particle-size distribution of the aerosol to increase, conversely, evaporation causes the mode to decrease.

\section{For cloud droplet}

The relative humidity at equilibrium is:

$$
R H=\frac{p_{s}}{p_{0}} \times 100 \%,
$$

where $p_{s}$ is the saturation vapor pressure above a particle at equilibrium (around a curved liquid droplet); $p_{0}$ is the saturation vapor pressure (flat surface of the same liquid).

The Kelvin equation for saturation vapor pressure above a curved surface is [14]:

$$
\ln \frac{p_{s}}{p_{0}}=\frac{2 \sigma M}{R_{1} T \rho r_{\rho}},
$$

where $\sigma$ is surface tension of droplet; $m$ is the molar mass; $R_{1}$ is the molar gas constant; $\rho$ is the density of liquid; $r_{\rho}$ is the droplet radius.

For PM 2.5

Deposition: Deposition is the process by which aerosol particles collect or deposit themselves on solid surfaces, decreasing the concentration of particles in air. Mechanisms for deposition are most effective for either very small or very large particles. Very large particles will settle out quickly through settling or impact process. While Brownian diffusion has greatest influence on small particles, e.g., the amount of PM 2.5 in air [15]. Deposition velocity is defined from [14]:

$$
F=v c,
$$

where $F$ is the flux density; $v$ is the deposition velocity; $c$ is the concentration. In gravitation, $v$ is the settling velocity due to gravity-induced drag.

Deposition due to Brownian motion obeys both Fick's first and second laws. The resulting deposition flux is defined as [14]:

$$
J=n \sqrt{D / \pi t}
$$

where $J$ is deposition flux; $n$ is the initial number density; $D$ is the diffusion constant; $t$ is time. This can be integrated to determine the concentration at each 
moment of time.

\section{The New Dynamic Equations of Aerosol in Air}

Summing up the results in section 5,6 , and 7 , we have

$$
\begin{aligned}
& \boldsymbol{\rho}_{a} \boldsymbol{a}_{a}-\boldsymbol{\rho}_{s} \boldsymbol{a}_{s}=\nabla \cdot \boldsymbol{p}, \\
& \frac{\partial \boldsymbol{\rho}_{s}}{\partial t}+\nabla\left(\boldsymbol{\rho}_{s} \delta \boldsymbol{u}\right)=0,(\text { for } \delta \boldsymbol{u} \neq 0) \\
& \frac{\partial \boldsymbol{\rho}_{s}}{\partial t}+\nabla\left(\boldsymbol{\rho}_{s} \boldsymbol{u}\right)=0,(\text { for } \delta \boldsymbol{u}=0) \\
& \frac{\partial \boldsymbol{\rho}_{a}}{\partial t}+\nabla\left(\boldsymbol{\rho}_{a} \delta \boldsymbol{u}\right)=0,(\text { for } \delta \boldsymbol{u} \neq 0) \\
& \frac{\partial \boldsymbol{\rho}_{a}}{\partial t}+\nabla \cdot\left(\boldsymbol{\rho}_{a} \boldsymbol{u}\right)=0,(\text { for } \delta \boldsymbol{u}=0) \\
& \frac{\partial\left[\rho_{s} \boldsymbol{u}_{s}\right]}{\partial t}+\nabla \cdot\left[\left(\boldsymbol{\rho}_{s} \boldsymbol{u}_{s}\right) \delta \boldsymbol{u}\right]=0,(\text { for } \delta \mathbf{u} \neq 0) \\
& \frac{\partial\left[\boldsymbol{\rho}_{s} \boldsymbol{u}_{s}\right]}{\partial t}+\nabla \cdot\left[\left(\boldsymbol{\rho}_{s}\right) \boldsymbol{u} \cdot \boldsymbol{u}\right]=0,(\text { for } \delta \boldsymbol{u}=0) \\
& \frac{\partial\left[\boldsymbol{\rho}_{a} \boldsymbol{u}_{a}\right]}{\partial t}+\nabla \cdot\left[\left(\boldsymbol{\rho}_{a} \boldsymbol{u}_{a}\right) \delta \boldsymbol{u}\right]=0,(\text { for } \delta \boldsymbol{u} \neq 0) \\
& \frac{\partial\left[\boldsymbol{\rho}_{a} \boldsymbol{u}_{a}\right]}{\partial t}+\nabla \cdot\left[\left(\boldsymbol{\rho}_{a}\right) \boldsymbol{u} \cdot \boldsymbol{u}\right]=0,(\text { for } \delta \boldsymbol{u}=0) \\
& \frac{\partial(\delta E)}{\partial t}+\nabla \cdot[\delta E \cdot \delta \boldsymbol{u}]=0,(\text { for } \delta \boldsymbol{u} \neq 0) \\
& \frac{\partial(\delta E)}{\partial t}+\nabla \cdot[\delta E \cdot \boldsymbol{u}]=0,(\text { for } \delta \boldsymbol{u}=0)
\end{aligned}
$$

The above vector Equations (8-1)- $(8-6)_{\mathrm{A}}$, supplemented by corresponding scientific laws for aerosol and air in Section 7 , form the new dynamic equations

of aerosol in air. It is a set of non-linear partial differential equations with unknown functions of $\boldsymbol{\rho}_{s}, \boldsymbol{\rho}_{a}, \boldsymbol{u}_{s}, \boldsymbol{u}_{a}, p, T$.

\section{Comparison and Features}

- Comparison with traditional general governing dynamic equation of aerosol (1-1).

The new general governing dynamic equation of aerosol in air has features: 1 , it belongs to certain type; 2 , it emphases the effect of buoyancy in derivation of equilibrium equation and on potential energy; 3 , it simplifies the effect of shape, size, and distribution of particles of aerosol on the calculation of motion of aerosol suspending in air for the case of $\delta \boldsymbol{u}=\mathbf{0}$. In which the aerosol particles follow the trace of air motion, and at least the wind speed equation of a point in air has been found [5]. 


\section{Conclusion}

The motion of aerosols in air is a two-phased flow problem. A model-in-model, in which a sub-model of control volume with smooth surface filled with aerosol particles, involved in a box with lengths $\mathrm{d} x, \mathrm{~d} y$, and $\mathrm{d} z$ approaching to zero and filled with air is used for study. Both box and sub-model are wrapped by "zero-weighted membrane". The new dynamic equations of aerosol in air are derived by equilibrium of buoyancy, gravity and pressure, together with conservation laws of mass, momentum and energy via Reynolds transport theorem and supplemented by corresponding scientific laws for related properties of air and aerosols. The new dynamic equations of aerosol in air of certain type is a set of non-linear partial differential equations involved unknown functions of mass density $\boldsymbol{\rho}_{a}, \boldsymbol{\rho}_{s}$, pressure $p$, wind speed $\boldsymbol{u}_{a}$, aerosol speed $\boldsymbol{u}_{s}$, and temperature $T$. It has features: 1 , it belongs to certain type; 2 , it emphases the effect of buoyancy in equilibrium and potential energy, and the Archimedes principle of buoyancy is firstly extended to lateral directions based on logical deduction, the phenomenon of stirring a glass of oil-water mixture and the recorded of Hurricane Isabel (2003) from space station. The later shows the evidence of existence of lateral buoyancy; 3 , the mass densities of air and aerosol of a point in our model are varied in different directions due to traction and are treated as vectors, and they have been used in the calculation of lateral buoyancy.

\section{References}

[1] Elgarayhi, A. (2002) Solution of the Dynamic Equation of Aerosols by Means of Maximum Entropy Technique. Journal of Quantitative Spectroscopy \& Transfer, 75, 1-11.

[2] Williams, M.M.R. and Loyalka, S.K. (1991) Aerosol Science: Theory and Practice with Special Applications to Nuclear Industry. Pergamon Press, New York.

[3] Frieder, S.K. (1977) Smoke, Dust, and Haze, Fundamental Behavior. Wiley, New York.

[4] Hinds (1999) p.49, Reference [19] of https://en.wikipedia.org/wiki/En/aerosol/physics/2017-05-13

[5] Yun, T.-Q. (2016) Wind Speed Equation of a Point in Air. Fundamental Journal of Modern Physics, 9, 57-64.

[6] Yun, T.-Q. (2016) Existence and Solution of Wind Speed Equation of a Point in Air. British Journal of Mathematics and Computer Science, 17, 1-5. Article no. BJMCS 27054.

[7] Yun, T.-Q. (2017) Wind Speed Equation of Circular Cyclone. British Journal of Mathematics \& Computer Science, 20, 1-8. Article No. BJMCS.31414.

[8] Yun, T.-Q. (2017) Solution of Wind Speed Equation of Circular Cyclone and Its Application. British Journal of Mathematics and Computer Science, 21, 1-13. https://doi.org/10.9734/BJMCS/2017/33249

[9] https://en.wikipedia.org/wiki/En/tropicalcyclone/2017-07-01

[10] https://www.chemeurope.com/ReynoldsTransportTheorem/2017-07

[11] https://en.wikipedia.org/wiki/ReynoldsTransportTheorem/2017-05 
[12] Editorial Group (1979) Hand Book of Mathematics. High Educational Publishers, Beijing, 446 p. (in Chinese).

[13] Clapeyrong, E. (1834) Memcire sur la puissance motrice de la chuleur”. Journal de I'Ecole Polytechnique, XIV: 153-90. (in French)

[14] https://en.wikipedia.org/wiki/Aerosol/physics/2017-06-18

[15] Seinfeld, J. and Pandis, S. (2006) Atmospheric Chemistry and Physicss: From Air Pollution to Climate Change. 2nd Edition, John Wiley \& Sons, Inc., Hoboken, New Jersey, ISBN: 0-471-72018-6. 